\title{
Numerical Simulation of the Two-Phase Sloshing with Different Densities in A2D Rectangular Tank under Lateral Excitement
}

\author{
Asad Mirahky ${ }^{1}$, Fereydoon Pavir ${ }^{2 *}$ and Mohamad Reza Ansari ${ }^{2}$ \\ 'Faculty of Mechanical Engineering, Tarbiat Modares University, Tehran, Iran; \\ a.mirahky@yahoo.com,mra_1330@modares.ac.ir \\ ${ }^{2}$ Mechanical Engineering Faculty, Sharif University of Technology, Tehran, Iran; \\ pavir@mech.sharif.ir
}

\begin{abstract}
The sloshing of liquid is occurred in a large variety of applications like reservoir containing liquid fuel, Liquefied Natural Gas (LNG), water and etc. In this paper, the critical frequency was found by comparing exciting frequency. The effects of density enhancement on decreasing the amplitude of produced waves and also the pressure imposed on the tank was investigated for various amounts of density. The critical frequency was found by comparing the exciting frequencies of the tank. The gauge pressure imposed on the roof and wall also was investigated in different points. In this paper, the critical areas on where the maximum pressure was applied, is shown. Upper phase density enhancement up to a special point was effective in reduction of sloshing and pressure wave altitude but as this enhancement increases, hydrostatic pressure goes up. The optimum density of the gas phase is $p^{\mu}=200 \frac{\mathrm{kg}}{\mathrm{m}^{3}}$ that reduces the interface sloshing and does not increase the pressure any more.
\end{abstract}

Keywords: Gas Phase, Resonance, Sloshing, Turbulent Flow

\section{Introduction}

The sloshing of liquid is occurred in a large variety of applications like reservoir containing liquid fuel, Liquefied Natural Gas (LNG), water and etc. If the natural frequency of the liquid is close to the exciting frequency of the tank the motion of liquid in a tank can cause large loads on it 2 . In general, the amplitude of the produced waves depends on the amplitude and frequency of excitement, depth of liquid, fluid characteristics and the geometry of the tank. These parameters have a direct effect on dynamic stability and performance of the tank. Investigation of interface in a container at a specific time resulted to the stability and pressure upon the rim. Formed waves in a container can cause instability and destruction of the container. Ibrahim published a review book from previous works. This book is about analytical results for a variety of different geometries and tanks excitation?
$\operatorname{In}$ conducted analytically and experimentally some research on the surface sloshing in a rectangular tank with an infinite roof for nonlinear, irrational flow by modal method. In their theory, a slight discrepancy was observed between experimental results and their modal method in an exciting frequency far from natural frequency of the tank and also they showed resonant modes in the $\operatorname{tank}$. Investigated the effect of the increasing upper phase density on fluids sloshing by finite volume method through Open FOAM software while external excitation frequencies were far from the natural frequency of the tank for non-rotational flow. They concluded that sloshing interface will be lowered by increasing density of the gas phase.

Using analytical methods and far from the resonance zone investigated the effect of increasing the upper phase density assuming the fluids to be irrational on changing the amplitude of the produced surface waves and showed 
that the density of the gas phase can be used to control the sloshing interfacel. Simulated sloshing in tank containing two fluids by considering the turbulence effects and by using FDM. In their study some simplifications were assumed including the fluids to be non-compressible and also not mixable non-compressible.

Inflycaptured the interface by the VOF method and normalizing the variable diagram with an unstructured grid. They excited the tank by the first natural frequency and achieved acceptable result in good agreement with experimental. Investigated the pressure distribution for two cases: with and without baffle in a rectangular tank using both the experimental and numerical approaches. They also made inquiries about the effect of baffles on the interface wave. In ${ }^{12}$ simulated the wave interface under different excitement frequencies and specified the frequency caused the resonant state in the tank. Applied some CFD methods like FEM1, FDM2 and FVM3 on their model resulting in more accurate result for the FVM method. In ${ }^{13}$ have found the numerical results of sloshing in rectangular tank under the random excitement by FEM According to studies carried out in the field of sloshing, gas and liquid interfaces were investigated more than the pressure in the tank. Most of the previous research have been done far from resonant reign in which sloshing is also not considered to be turbulent. In this paper, sloshing investigation in resonance state for turbulent flow carried out.

\section{Natural Frequencies of the System}

Natural frequency or natural mode are obtained from solving the potential equation for liquid phase, neglecting the gas phase on the condition of no excitement (the continuity equation is considered). Zero boundary condition excitement on the tank meaning $\frac{\partial \emptyset}{\partial \mathrm{n}}=0$ in the $S_{0}$ should be consider ( $\emptyset$ is potential equation). The center of coordinate is on free non sloshing surface, Figure 1, which $Q_{0}$ and $S_{0}$ are the fluid liquid volume and wet area of the tank, respectively. $\sum_{0}$ stands for the free surface area of liquid. The fluids in the tank are considered as non-turbulent and therefore the potential equation can be solved for the tank. The boundary condition for the case with no excitement could be applied as Newman's B.C $\left(\frac{\partial \emptyset}{\partial n}=0\right)$ at boundaries. Total volume of the fluid remains constant, so $\int_{z_{e}} \xi d x d y=\mathbf{0}$. $z=\xi(x, y, t)$ denotes the height of free surface.

The equations of free surface height and the potential flow equation are 2 .

$$
\begin{aligned}
& \xi(x, y, t)=f(x, y) \exp (i \omega t) \\
& \phi(x, y, t)=\frac{i g}{\omega} \varphi(x, y, t) \exp (i \omega t)
\end{aligned}
$$

In which $\omega$ is the natural frequency of the system, $\phi$ is potential equation and $\xi$ is?.Equation 3 shows the potential equation for $2 \mathrm{D}$ condition. Solving this equation by applying the B.C., natural frequencies of the system could be obtained as shown in equation 4 . This equation gives countless natural frequencies for a tank by just considering the liquid phase i.e. ignoring the gas phase. The upper phase density and its effect on the other phase's dose not satisfy Laplacian equation. In the majority of cases in which the gas phase density is low, the results show good accuracy.

$\frac{\partial^{\mathbf{2}} \varphi}{\partial^{2} x}+\frac{\partial^{2} \varphi}{\partial^{2} y}=\mathbf{0}$

$\omega_{i}=\sqrt{g \frac{\pi i}{l} \tanh \left(\frac{\pi i h}{l}\right)}$

In equation $4, \boldsymbol{h}$ is the height of the free surface and $l$ is width of the tank. Equation 4 is derived by the assumption of non-circulating flow and low amplitude waves. None of the characteristics of the fluid is included in relation 4 and consequently it is valid for all the fluids, but depend on the fluid type, the amplitude of the produced waves may be differ shown in Figure 1.

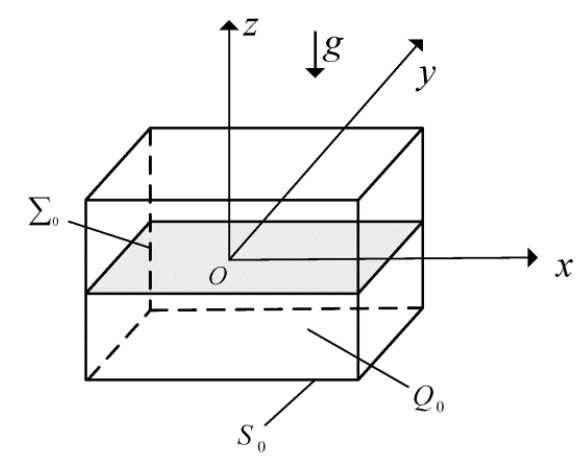

Figure 1. Rectangular tank with liquid fluid filling $Q_{0}$,free surface of and wet area. 


\section{General Problem Setup}

For this studied case, surface waves of water are produced by the excitement of $x=A \sin \left(w_{h} t\right)$ where A is the excitement amplitude and $\omega_{\boldsymbol{h}}$ is the excitement frequency of the rectangular tank containing two phases of liquid and gas.

The tank is divided into two parts. The bottom side is filled with water $\left(\rho_{1}\right.$ water $\left.=1000 \mathrm{~kg} / \mathrm{m}^{\uparrow} 3\right)$ and the upper side is filled with gas with various densities, i.e. $\rho_{\text {gas }}=1.3,50,100,200, \frac{800 \mathrm{~kg}}{\mathrm{~m}^{3}}$.

The dimensions of the tank are $\mathrm{L}=1.73 \mathrm{~m}$ (width) and $\mathrm{H}=1.05 \mathrm{~m}$ (height). The initial free surface height is $\mathrm{h}$ from the bottom of the tank the pressure was measured in four points (probe 1-4) in order to evaluate the numerical simulation shown Figure 2. The height of liquid in FS line was measured and compared with experimental results.

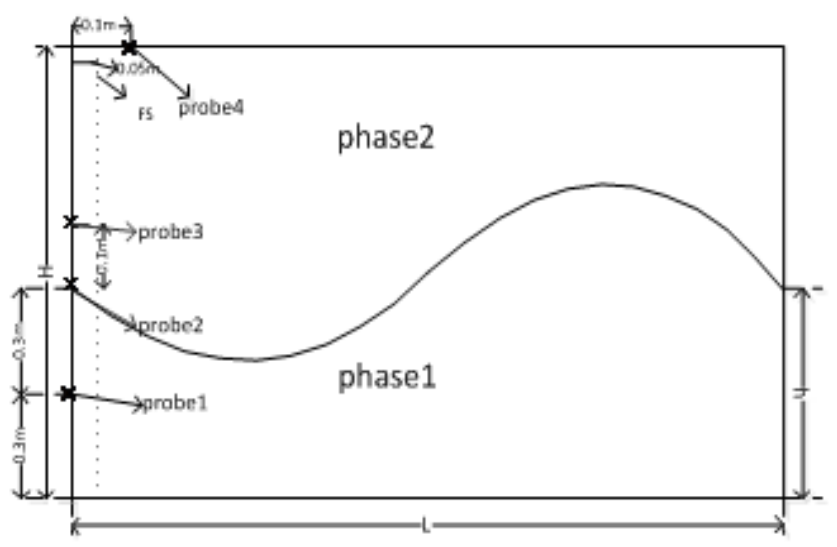

Figure 2. 2D tank with pressure probe.

\section{Numerical Solution Method}

\subsection{Computational Tools}

This equation has not a general analytic solution and is solved by numerical method.

In equation 4 , a tank has a large number of natural frequencies leading to a disaster in a frequency near the natural frequency of the system called resonance phenomenon. When resonance occurred, the amplitude of the fluid waves would be increased rapidly and lead to a complicated turbulent flow. The simulations were done based on LES/ model.

Navier-strokes equations govern the two phase's flow for two dimensions and are discretized by FVM. The VOF method is used to capture the interface. Simulation is done by Open FOAM software. The software includes different solvers for various fields of fluid mechanics. In this paper, Inter DyM Foam solver is used. This solver is compatible for non-compressible multi-phase flows with a dynamic mesh. It is based on FVM and uses PISO algorithm to solve the coupling between pressure and velocity fields. Considering all the discretization methods here, "backward" (second order, implicit) time integration, "Gausslinear" (second order, Gaussian integration with linear interpolation) discretization for the gradient, divergence and Laplacian operators ("corrected", for the later).

To avoid the numerical instability, maximum of Courant number is set 0.5 . Open FOAM has the capability to adjust the time step by considering maximum Courant number. The $2 \mathrm{D}$ rectangular geometry was meshed by Cartesian method. Table 1 lists the number of grids in Cartesian system. For these three cases the liquid height was measured in FS as can be seen in figure2. Grid independency is provided on Figure 3. For reasonable accurate results, grid number of $86^{\star} 52$ was selected shown in Table 1.

Table 1. Grids distribution

\begin{tabular}{lccc}
\hline & $\mathbf{x}$ & $\mathbf{y}$ & total \\
\hline GRID 1 & 86 & 52 & 4472 \\
GRID 2 & 43 & 26 & 1118 \\
GRID 3 & 27 & 17 & 459 \\
\hline
\end{tabular}

The VOF method was used for obtaining the interface between the gas and liquid phases in the tank $k^{4,13}$. This method gives acceptable results for sloshing phenomenon for a condition that the tank is full of two phasest shown in Figure 3. Since a number named volume fraction is attributed to each cell, which shows the phase fraction in each cell, an equation is added to momentum and continuity to calculate this coefficient which is shown in the following equations:

$\rho=\alpha \rho_{l}+(1-\alpha) \rho_{g}$

$\mu=\alpha \mu_{l}+(1-\alpha) \mu_{g}$

$a$ is a volume fraction and scalar parameter, this parameter is a value between $0-1$. Cells with the value $0<a<1$ define the gas and liquid interface. Density and viscosity can be obtained by equation of (5) and (6) for momentum equation. 
Dynamic mesh was applied to solve this problem, because it forces the results converge rapidly. The solution time of solving problem by dynamic mesh is less than other methods using boundary condition ${ }^{17-19}$.

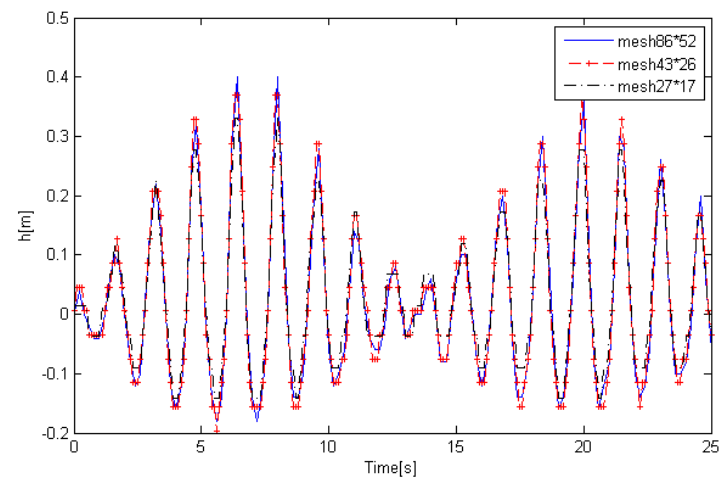

Figure 3. Liquid height in FS.

\section{Results}

\subsection{Prediction the Interface}

In this study in order to validate the performed simulations, numerical results were compared with experimental results. Numerical results for two experimental cases in different heights and excitement were done and that shown below.

- Liquid height $0.6 \mathrm{~m}$, the excitation frequency 4.1817 $\mathrm{rad} / \mathrm{s}$, the amplitude excitement $0.03 \mathrm{~m}$

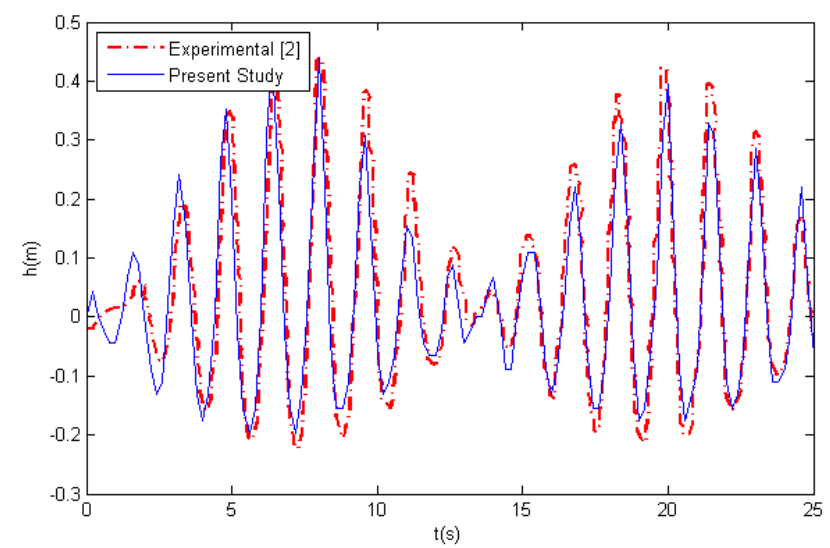

(a)
- Liquid height $0.5 \mathrm{~m}$, the excitation frequency 4.7204 $\mathrm{rad} / \mathrm{s}$, the amplitude excitement $0.045 \mathrm{~m}$

Height liquid in FS for two case of top is shown in Figure $4 \mathrm{a}$ and Figure $4 \mathrm{~b}$. the numerical and experimental results have good agreement shown in Figure 4.

In order to verify numerical results, two cases of works were selected ${ }^{2}$. In both cases the characteristics of container are identical. i.e. width $\mathrm{L}=1.73 \mathrm{~m}$, height $\mathrm{H}=1.05 \mathrm{~m}$, fluid height $\mathrm{h}=0.6 \mathrm{~m}$, and densities of liquid and gas are $1000 \mathrm{~kg}$ and $1.3 \mathrm{~kg}$, respectively.

$$
m^{3} \quad \mathrm{~m} 3
$$

The first and the second cases have frequency and amplitude of $\frac{\omega}{\omega_{1}}=1.11 \quad\left(\omega=4.1817 \frac{\mathrm{rad}}{\mathrm{s}}\right)$, $\frac{A}{L}=0.0173 \mathrm{~m} \quad(A=0.03 \mathrm{~m})$ and $\frac{\omega}{\omega_{1}}=1.283$ $\left(\omega=4.8334 \frac{\mathrm{rad}}{\mathrm{s}}\right), \frac{A}{L}=0.01445 \mathrm{~m} \quad(A=0.025)$,

respectively. The results of two cases are demonstrated in Figures 4 and 5. In both figures the surface elevation of fluid was measured at the left rim (FS) in distance of $5 \mathrm{~cm}$.

The critical condition in a container occurs when amplitude of produced waves would be increased. Therefore, instability and collision against the rim and container roof is anticipated. This phenomenon, according to the fourth equation, is occurred when the natural and stimulant frequencies are equal.

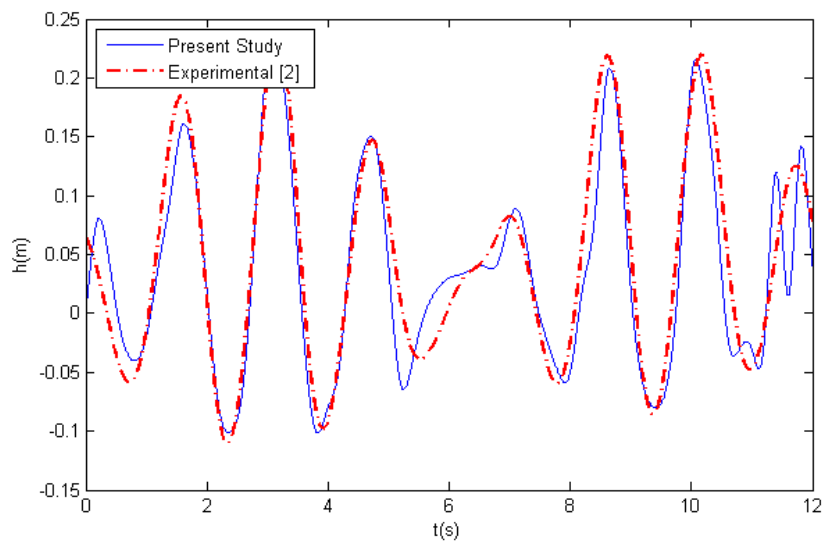

(b)

Figure 4. Comparison of numerical results with experimental (a) $\omega=4.1817 \frac{\mathrm{rad}}{\mathrm{s}}, A=0.03 \mathrm{~m}$;

(b) $\omega=4.8334 \frac{\mathrm{rad}}{\mathrm{s}}, \mathrm{A}=0.025 \mathrm{~m}$ 


\subsection{Comparison of Laminar and Turbulent Flow}

In the tanks excitation frequencies close to the natural frequency of the system, waves in the interface would grow rapidly, hit the roof and cause turbulent flow. Numerical result for laminar and turbulent flows under the excitation of $0.03 \sin (4.1817 \mathrm{t})$ were compared with experiment and results are shown in Figure 5. For turbulent flows results are more accurate. However, in this case, since the excitation frequency is not the same as the system natural frequency, sloshing is calm. In the cases including resonance, turbulent flow has more accurate result compared to experiment.

Compare model turbulence are done in Figure 6 that just LES model has accurate results and two other model $\mathrm{k}$-epsilon and k-omega physics is to misread.

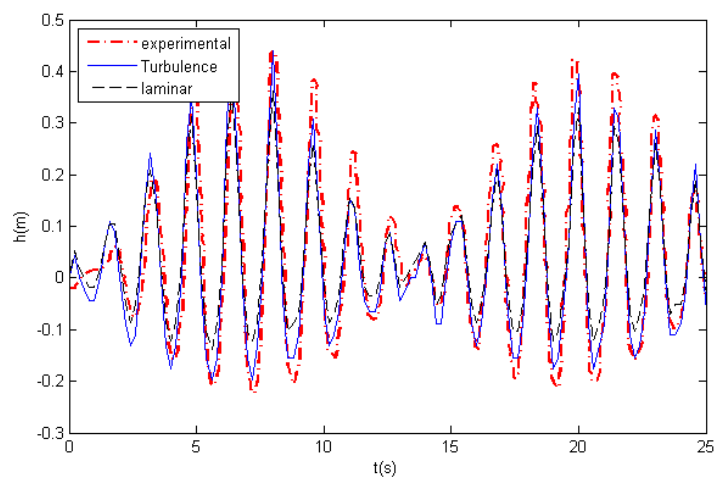

Figure 5. Liquid height in FS in the turbulent steady state and compared with experimental.

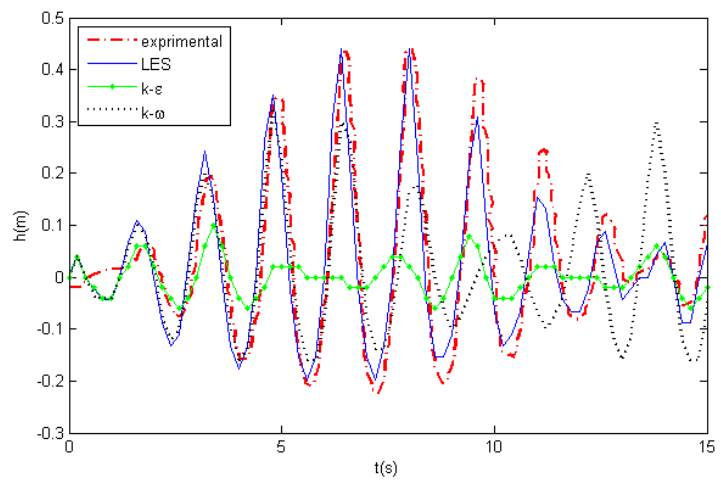

Figure 6. Different models of turbulent flow in the liquid height in FS compared with experimental.

\subsection{Critical Excitement Frequency} Critical frequency equal to the natural frequency through which resonance occurs, the wave amplitude reaches container roof and causes instability and hits roof and wall tanks. The worst case occurs when the excitation frequency is equal to the natural frequency of the tank.

Results related to different excitement frequencies with gas phase density of $\rho^{\mathrm{u}}=1.3 \frac{\mathrm{kg}}{\mathrm{m}^{3}}$ and similar excite amplitude $(\mathbf{A}=\mathbf{0 . 0 3} \mathrm{m})$ is shown in Figure 7. It can be inferred that at frequencies lower than the first natural frequency of the wave amplitude initially increases and then decreases, this trend will continue. By increasing frequency excitement reaching the first natural frequency, resonance occurs. Wave amplitude rises rapidly and tank tops collide and cause instability. At frequencies greater than first natural frequency is equal the frequencies lower than first natural frequency. Toward the second natural frequency, high rate of stimuli does not let high height waves to be formed. Other greater frequencies along with high speed container keep the same pattern. For a worst case frequency stimulation of the tank, is the first natural frequency excitemented

The highest amplitude of formed stimulant waves has equal frequency to the first natural frequency $\left(\omega_{1}\right)$. One way to reduce fluid wave amplitude in the container is to increase gas phase density. Fluid height in the FS when upper phase density is $\rho^{u}=1.3,50,100,200,800 \frac{\mathrm{kg}}{\mathrm{m}^{3}}$ respectively by this excitement $0.03 \sin \left(\omega_{1} t\right)$ was measured and the results are shown in Figure 8. As phase density is incremented, amplitude of produced waves would be reducing. Consequently, the first natural frequency is not the container natural frequency when the upper phase density increases. Because of the greater density of the gas phase, the amplitude of the wave is not generally incremental and increase, decrease operating. There is no absolute incremental and the amplitude variation is oscillatory. Increment of phase density reduce the waves' amplitude intensely and prevent fluid to reach the top as well as no collision occurs. With the incremental phase density, sloshing reduce continuingly which lead to very small oscillations on the interface for density of $\rho^{u}=800 \frac{\mathrm{kg}}{\mathrm{m}^{2}}$. 


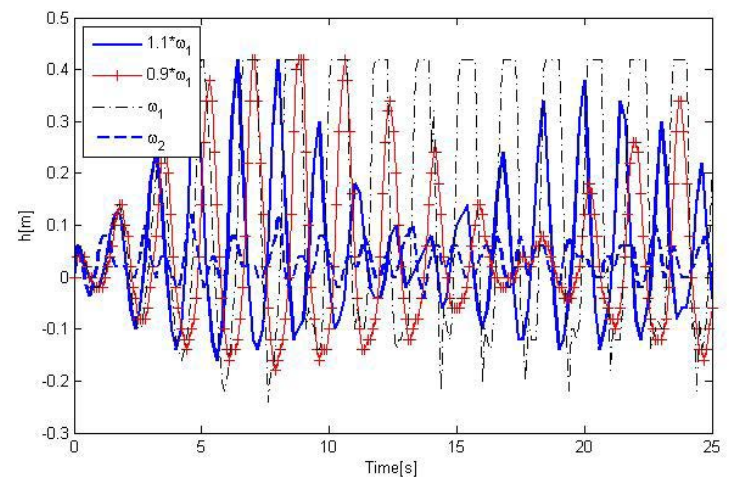

Figure 7. Comparing the height of liquid in the FS for different excitation frequencies.

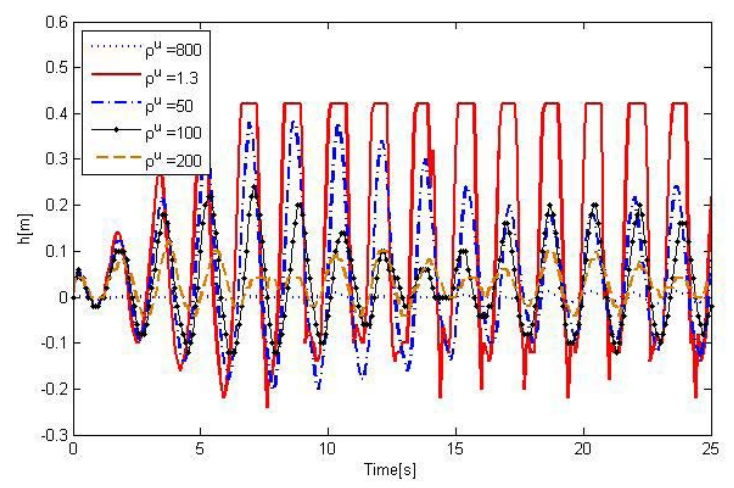

Figure 8. Comparing the height of liquid in FS for the different gas phase density.

\subsection{Pressure on Rim and Ceiling}

Produced waves with high amplitude in container and their collision to rim and ceiling exert a high pressure. These high pressures are mostly impassive and increase instability and destruction probability in the container. Gauge pressure is used to compare pressures. As gas density increased, the amplitude of formed waves would be reduced. And is also expected to reduce the pressure on the walls and ceiling. The pressure on four distinct points of container is measured are shown in Figure 9. All investigated states have similar amplitude $(A=0.03 \mathrm{~m})$ and excitement frequency $\omega=\omega_{1}=3.7673$. The excitement of container is equal to $x=A \sin (w t)$.

The simulation results of the first probe are shown in Figure 9a. Most oscillations correspond to the density of $\rho^{u}=1.3 \frac{\mathrm{kg}}{\mathrm{m}^{3}}$. As the gas phase density increased, the oscillations would be decreased. However, mean value of gauge pressure increases with growth of gas density and in probe1, maximum pressure in the gas density is $\rho^{u}=800 \frac{\mathrm{kg}}{\mathrm{m}^{3}}$. At the second probe, shown in Figure 9b, the tangential place of left rim probe on liquid surface causes the probe to become more sensitive to the sloshing. Thus, the amplitude of pressure waves increases in this probe. In probe2, except for $\rho^{u}=800 \frac{\mathrm{kg}}{\mathrm{m}^{\mathrm{s}}}$ in three other gas density, sloshing is more and do not have more different in amplitude pressure. The third probe follows the same procedure probe1 and 2, as the pressure wave's amplitude in density of $\rho^{u}=1.3 \frac{\mathrm{kg}}{\mathrm{m}^{3}}$ are more than other states are shown in Figure 9c.

The pressure corresponding to the fluid height was decreased with the increase of probe -position. Therefore, the pressure impacts corresponded to collision of fluids would be increased. Figure $9 \mathrm{~d}$ is related to the forth probe. It shows that the shock waves hit the ceiling of container which causes instability and destruction. These waves enforce greater forces with greater scales. The waves are shocking with very high amplitude. The amplitude of shock waves of this probe and their exerted pressure are more critical in comparison to other probes 1,23 . The most critical point in the container is the place of probe 4 .

By increasing the density of the gas phase the continuous sloshing is reduced. But with the increasing density of the gas-phase partial pressure is increased. Here it can be seen; in the gas phase $\rho^{\mathrm{u}}=200 \frac{\mathrm{kg}}{\mathrm{m}^{3}}$ amplitude wave pressure decreases and means pressure does not increase too much. The container in the figure 1 was undergone three distinct frequencies with the same amplitude and the upper phase density of $\rho^{u}=1.3 \frac{\mathrm{kg}}{\mathrm{m}^{3}}$.

Gauge pressures in critical point (probe4) for three different frequencies are demonstrated in Figure 7. It can be inferred that the fluid reaches the container roof only at the first natural frequency. At this frequency, the wave exerts a high shocking pressure. In the two other cases, no pressure is exerted to the roof are shown in Figure 9 and 10. 

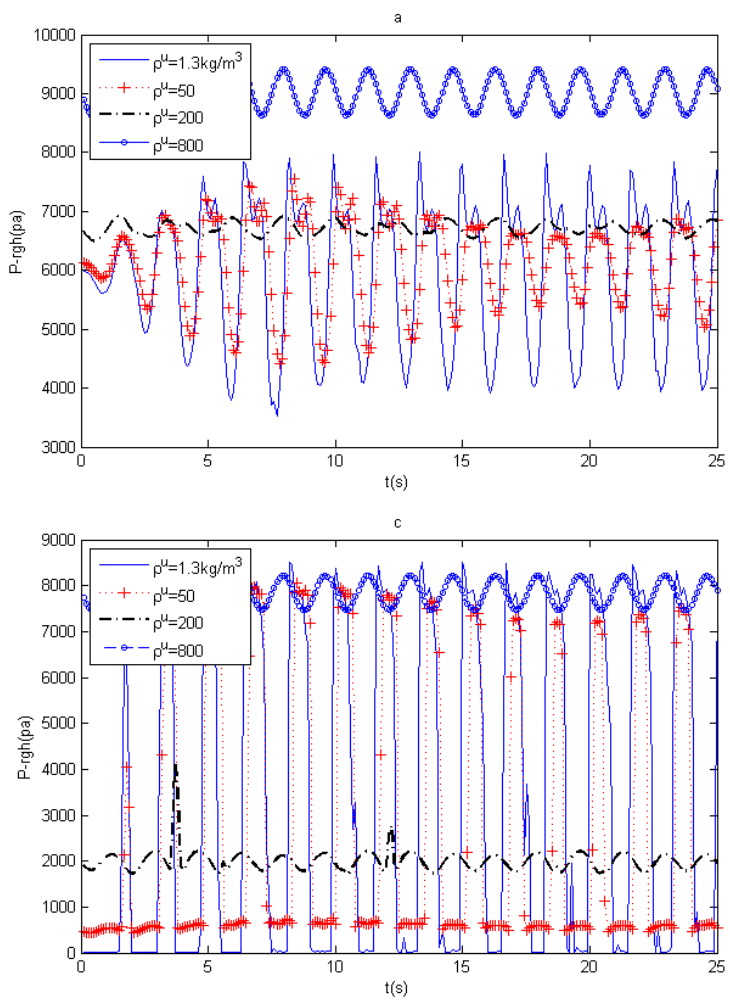
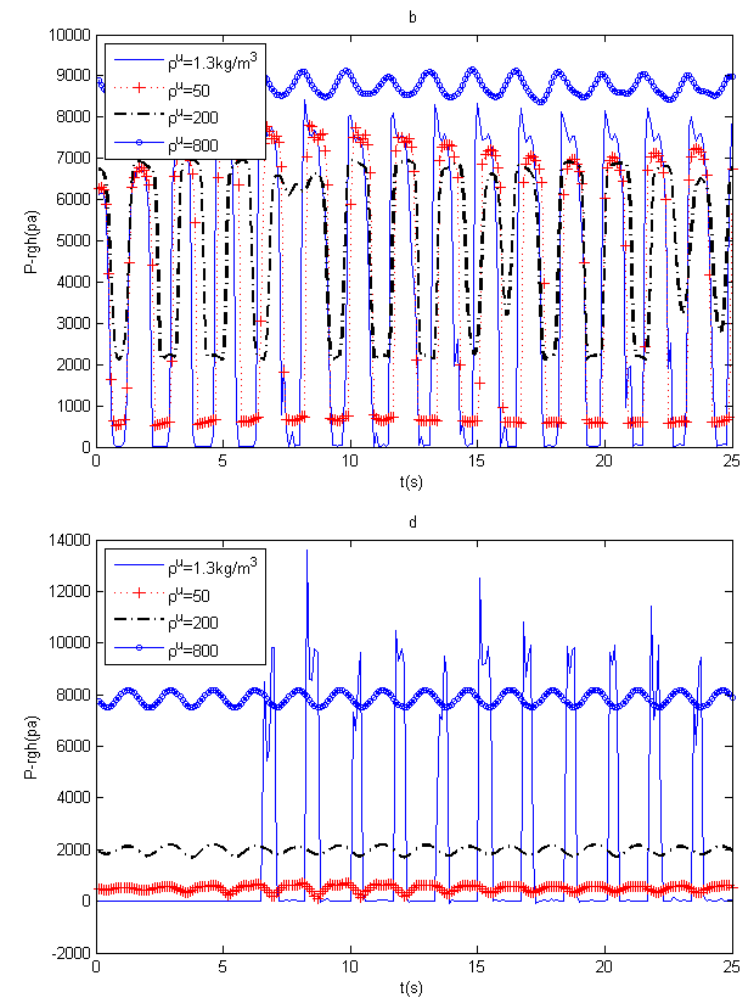

Figure 9. Gauge pressure in the first 25 seconds under the same excitation at four different probe of gas phase (a) Represents 1; (b) Indicates 2; (c) Indicator 3; (d) 4 marks.

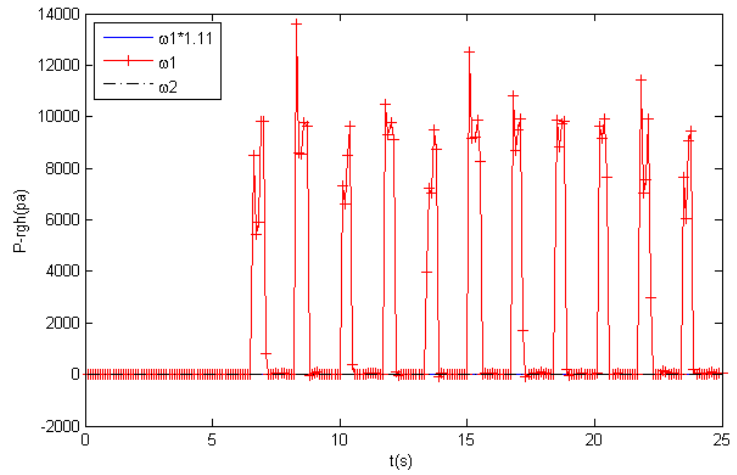

Figure 10. Gauge pressure for three different frequencies at marker 4.

\section{Conclusions}

In this study, sloshing interface and gage pressure by using Open FOAM software was obtained with the assumption of turbulent flow by LES model. Navier-Strokes equation terms were discretized in both phases by FVM method. VOF method was used to capture the interface. Resonance phenomenon may occur in case of equality between excitement and natural frequencies. The most destructive state is related to the case in which excite frequency is equal to the first natural frequency in tank. In fact, there is enough time for great amplitude waves to be formed in the first natural frequency but, the time shortens in higher natural frequencies as the container speeds up, so the amplitudes would be less. In case of equality between the first natural frequency and excite frequency, waves amplitude increase constantly till they reach the roof of container causing instability and destruction. In order to control the produced waves, one approach is to increase the upper phase density. This increase in upper phase density reduces the waves and pressure amplitude, but increasing density of the gas phase increase hydrostatic pressure. Optimum state for density gas phase to control the sloshing and decrease wave amplitude pressure is $\rho^{\mathrm{u}}=200 \frac{\mathrm{kg}}{\mathrm{m}^{3}}$, this density does not increase hydrostatic pressure more. 


\section{References}

1. Ibrahim RA. Liquid Sloshing Dynamics: Theory and Applications. New York: Cambridge University Press; 2005. p. 972.

2. Wang CZ, Khoo BC. Finite element analysis of two dimensional nonlinear sloshing problems in random excitations. Ocean Engineering. 2005; 32(2):107-33.

3. Akyilidiz H. A numerical study of the effects of the vertical baffle on liquid sloshing in two-dimensional rectangular tank. Journal of Sound and Vibration. 2012; 331(1):41-52.

4. Faltinsen M. Hydrodynamics of High-Speed Marine Vehicles. New York: Cambridge University Press; 2005.

5. Ibrahim RA, Pilipchuk VN, Ikeda T. Recent advances in liquid sloshing dynamics. Applied Mechanical Review. 2001; 54(2):133-99.

6. Faltinsen OM, Timokha AN. An adaptive multimodal approach to nonlinear sloshing in a rectangular tank. Journal of Fluid Mechanics. 2001; 432:167-200.

7. Goni GJ, Mendoza GA, Cercos L, Gonzalez L. Two phase analysis of sloshing in a rectangular container with Volume Of Fluid (VOF) methods. Ocean Engineering. 2013; 73(15):208-12.

8. Ansari M, Firouz-Abadi R, Ghasemi M. Two phase modal analysis of non-linear sloshing in a rectangular container. Ocean Engineering. 2011; 38(11-12):1277-82.

9. Kim Y, Shin YS, Lee KH. Numerical study on slosh-induced impact pressures on three-dimensional prismatic tank s. Ocean Research. 2004; 26(5):213-26.

10. Jian PM, Yang WD. Numerical simulation of sloshing in rectangular tank with VOF based on unstructured grids. Journal of Sound and Vibration. 2007; 302(22):657-82.

11. Akyildiz H, Unal EN. Experimental investigation of pres- sure distribution on a rectangular tank due to the liquid sloshing. Ocean Engineering. 2005; 32(11-12):1503-16.

12. Rebouillant $S$, Liksonov S. Fluid-structure interaction in partially filled liquid container: A comparative review of numerical approaches. Computers and Fluids; 2010; 39(5):739-46.

13. Wang CZ. Finite element analysis of two-dimensional nonlinear sloshing problems in random excitations. Ocean Engineering. 2005; 32(2):107-13.

14. Hirt CW, Nichols BD. Volume Of Fluid (VOF) method for the dynamics of free boundaries. Journal of Computational Physics. 1981; 39(1):201-25.

15. Muzaferija S, Peric M. Computation of free surface flow using interface-tracking and interface-capturing methods. Nonlinear Water Wave Interaction Computational Mechanics. 1999; 183(1):59-100.

16. Kleefsman KT, Fekken G, Veldman AP, Iwanowski B, Buchner B. A volume-of-fluid based simulation method for wave impact problems. Journal of Computational Physics. 2005; 206(1):363-93.

17. Mehta HD, Rajesh P. optimal design of transformer using tournament selection based elitist genetic algorithms. Indian Journal of Science and Technology. 2015; 8(12):12-20.

18. Mashadi B, Mahmoudi-Kaleybar M, Ahmadizadeh P, Oveisi A. A path-following driver/vehicle model with optimized lateral dynamic controller. Lat Am J Solids Struct. 2014; 11(4):623-30.

19. Casadei GM. Dynamic-mesh techniques for unsteady multiphase surface-ship hydrodynamic [Master of Science Thesis] Mechanical Engineering; 2010.

20. Kazemi M. Buckling analysis of bi-modular laminated plates: A comparative study using new modified higher order theory. Indian Journal of Science and Technology. 2015; 8(17):175-81. 